\title{
Silk Fibroin Acts as a Self-Emulsifier to Prepare Hierarchically Porous Silk Fibroin Scaffolds through Emulsion-Ice Dual Templates
}

\author{
Jianchuan Wen, Jinrong Yao, Xin Chen, ${ }^{\oplus}$ and Zhengzhong Shao*๑
}

State Key Laboratory of Molecular Engineering of Polymers, Department of Macromolecular Science, Laboratory of Advanced Materials, Fudan University, Shanghai 200433, P. R. China

\section{Supporting Information}

\begin{abstract}
Silk fibroin (SF) has shown enormous potentials in various fields; however, application of SF in emulsion technology is quite limited. Here, we use SF as a selfemulsifier to form an oil-in-water $(\mathrm{O} / \mathrm{W})$ emulsion by emulsifying 1-butanol in SF aqueous solution. This showed that SF possessed strong surface activity to stabilize the $\mathrm{O} / \mathrm{W}$ emulsion without the need for any other surface-active agent until its solidification because of 1-butanol-induced conformational transition of SF to $\beta$-sheet. After freezing the preformed emulsions at $-20{ }^{\circ} \mathrm{C}$, robust three-dimensional porous $\mathrm{SF}$ scaffolds were prepared without the need for any further posttreatment. The evolution from the $\mathrm{O} / \mathrm{W}$ emulsion to porous scaffold formation under freezing was tracked, and an emulsion-ice dual template mechanism was proposed for scaffold formation, based on which SF scaffolds with controllable hierarchically porous structures were achieved by tuning the dispersed droplet volume fraction. Furthermore, SF scaffolds with hierarchical porosity showed significantly higher bioactivity toward L929 fibroblasts than that of SF scaffolds with mono macroporosity, highlighting the great asset of this hierarchically porous SF scaffold for broad applications in tissue engineering. Therefore, the strong surface-active characteristic of SF presented here, in addition to its distinct advantages, sheds a bright light on the application of SF in the vast range of emulsion technologies, especially in cosmetic-, food-, and biomedical-related areas.
\end{abstract}

\section{INTRODUCTION}

Tissue engineering provides a promising solution toward failure of tissues and/or organs arising from disease or other damages. ${ }^{1}$ The success of tissue engineering greatly relies on the construction of suitable scaffolds. ${ }^{1-4}$ Extracellular matrix (ECM) is an excellent example of a natural scaffold with an exquisite hierarchical architecture (a collection of macro-, micro-, and nanoscale features), which is able to offer an optimal environment for cells and therefore to maintain excellent biological functions. ${ }^{5,6}$ Previous studies have shown that biomimetic scaffolds designed with hierarchically porous structures showed better cellular functions and tissue regeneration than those of the conventional ones that have only macroporosity; however, materials used for such scaffolds were limited to only a few synthetic polymers. ${ }^{6-8}$ Therefore, it is highly alluring as well as challenging to develop more strategies to construct hierarchically porous scaffolds using biomacromolecules that have been widely used to make scaffolds due to their distinct advantages in bioactivity, biocompatibility, and biodegradability.

Silk fibroin (SF) from the core of Bombyx mori silkworm silk, a cheap and sustainable protein fiber with abundant supply through the mature sericulture industry flourishing in textile for centuries, has now shown vast progress in developing a wide range of biomaterials due to its excellent mechanical properties, biocompatibility, and biodegradability. ${ }^{9-16}$ The SF molecule consists of a heavy chain $(390 \mathrm{kDa})$ and a light chain $(25 \mathrm{kDa})$, which are connected by a disulfide link. ${ }^{17}$ The highly repetitive GAGAGS (G: glycine; A: alanine; S: serine) amino acid motifs constitute the primary structure of the heavy chain and is considered as the crystallizable segment, whereas the rest amino acid residues form the amorphous region. ${ }^{18}$ There are 12 hydrophobic crystalline domains distributed in 11 hydrophilic amorphous domains and 2 hydrophilic terminals, which make SF a natural amphiphilic multiblock copolymer. ${ }^{19-21}$ On the basis of this characteristic structure, the assembly behavior and structure of SF at the interface attract extensive attention; ${ }^{22-27}$ however, research on SF at the emulsion interface is still insufficient.

Currently, SF scaffolds are mainly prepared using particulate leaching (e.g., $\mathrm{NaCl}$ salt particles), gas foaming, lyophilization, or three-dimensional (3D) printing methods; however, these approaches either suffer from the need for tedious posttreatments or lack the ability to control the hierarchical structure of the pores. ${ }^{9-11,28}$ We previously developed a facile method to fabricate 3D porous SF scaffolds by simply freezing

Received: November 28, 2017

Accepted: February 13, 2018

Published: March 21, 2018 

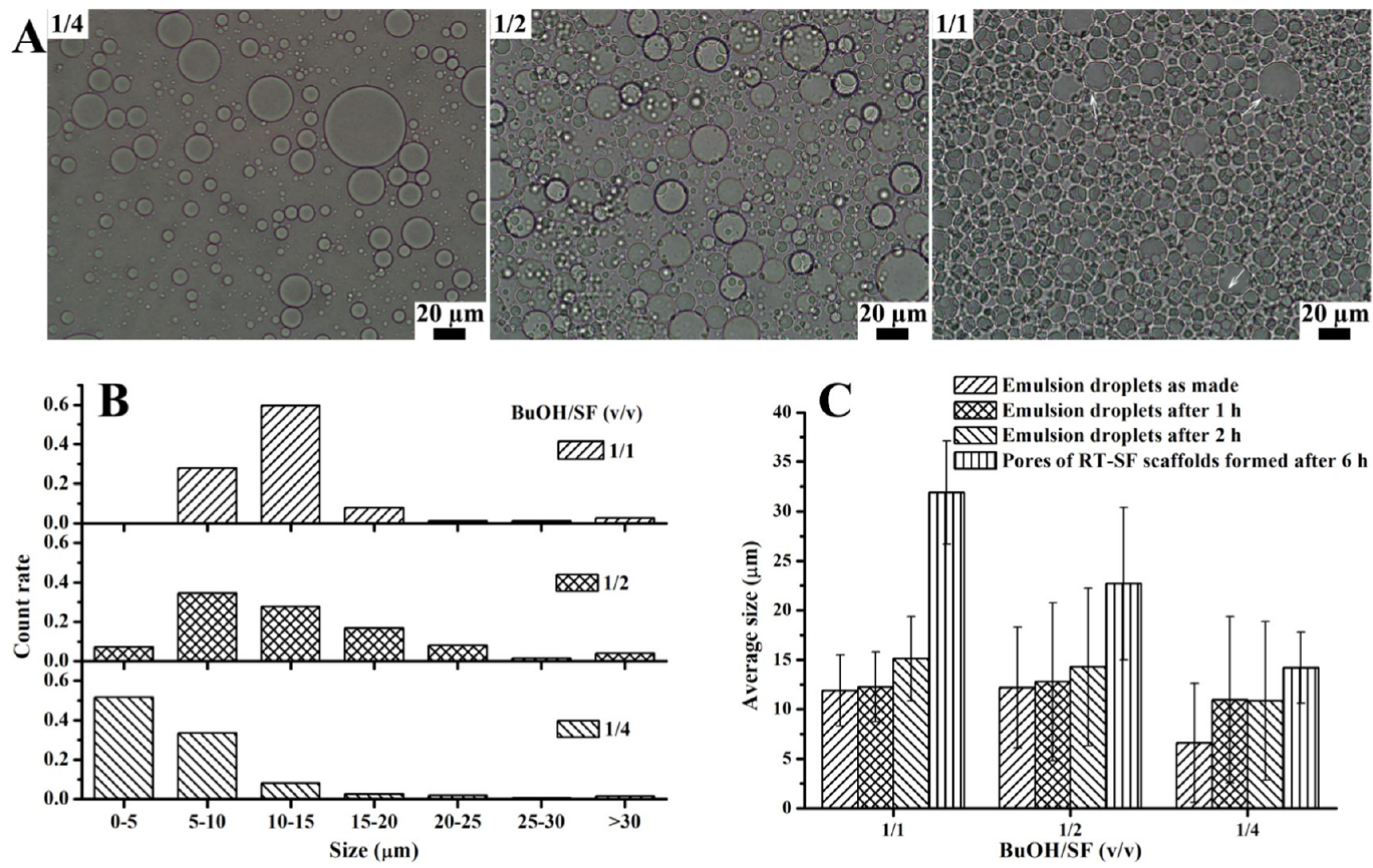

Figure 1. (A) Optical microscopic images and (B) emulsion droplet size analysis of $\mathrm{O} / \mathrm{W}$ emulsions prepared with $\mathrm{BuOH} / \mathrm{SF}$ of $1 / 4,1 / 2$, and $1 / 1$ (the arrows indicate slight deformation at the edge of the emulsion droplets) and (C) average droplet size of $\mathrm{O} / \mathrm{W}$ emulsions and average pore size of RT-SF scaffolds formed from these emulsions after different periods at room temperature.

the mixtures of 1-butanol and SF aqueous solution, which not only provided a flexible approach for gross shape control but also avoided the use of highly toxic chemicals and any further post-treatment (such as high-energy-consuming freeze-drying). ${ }^{29}$ To better understand the mechanism of scaffold formation of this technique as well as self-assembly behavior of SF at the emulsion interface, and therefore to achieve exquisite regulation of the porous structure of SF scaffolds, we investigated the oil-in-water $(\mathrm{O} / \mathrm{W})$ emulsions formed using 1butanol as the oil phase and SF aqueous solution as the water phase in the present study. It is expected that SF would act as a self-emulsifier able to stabilize the formed $\mathrm{O} / \mathrm{W}$ emulsions; thereafter, conformational transition of SF induced by 1butanol would lead to solidification of the emulsions, resulting in the formation of SF scaffolds. SF scaffolds formed via emulsions at room temperature (RT) or $-20{ }^{\circ} \mathrm{C}$ were investigated. On the basis of these studies, the scaffold formation mechanism of this technique and application of these SF scaffolds were explored. We expect that this study would provide important insight into preparing hierarchically porous SF scaffolds for broad applications in tissue engineering and applying SF, considering its strong surface-active characteristics and other distinct advantages, in the vast range of emulsion technologies, especially in cosmetic-, food-, biomedical-related areas.

\section{RESULTS AND DISCUSSION}

SF Self-Emulsified O/W Emulsions. When 1-butanol was added into a SF aqueous solution at room temperature, due to low solubility of 1 -butanol in water (i.e., 8.03 wt $\%$ at $20{ }^{\circ} \mathrm{C}$ ), ${ }^{30}$ phase separation occurred. After 2 days, a white film formed at the 1-butanol/water interface; thereafter, the SF solution in the sublayer gradually turned into a white SF gel in one more day (Figure S1A-C, see Supporting Information). On the basis of these observations and our previous research on behaviors of SF at the water-air interface, ${ }^{25}$ we believe that SF possesses superior surface activity because of its natural characteristic of an "amphiphilic multiblock copolymer" structure. Therefore, in the 1-butanol/SF solution system without stirring, SF chains would slowly diffuse from aqueous solution to the interface between water and 1-butanol and adsorb there to minimize the interface tension. SF chains at the interface would then rearrange to expose hydrophilic domains toward the aqueous phase whereas hydrophobic domains toward the organic phase, which consequently facilitated conformational transition of SF induced by 1-butanol to assemble and form a white film first at the interface due to the higher 1-butanol content around the interface. $^{31}$ Thereafter, conformational transition of SF in the bulk aqueous solution began to accelerate taking SF assemblies at the interface as nuclei, ${ }^{32}$ resulting in the formation of SF gel. On the other hand, when stirring $(400 \mathrm{rpm})$ was applied to the 1-butanol/SF solution mixture, an $\mathrm{O} / \mathrm{W}$ emulsion formed with 1-butanol droplets dispersed in the continuous SF solution. Eventually, the emulsion solidified into a scaffold in about $6 \mathrm{~h}$ at room temperature (RT-SF scaffold; Figure S1D,E, see Supporting Information). Apparently, stirring not only sheared 1-butanol into dispersed droplets but also accelerated SF's diffusion to and adsorption at the interface as well as matter exchange between the two phases, leading to faster molecular 

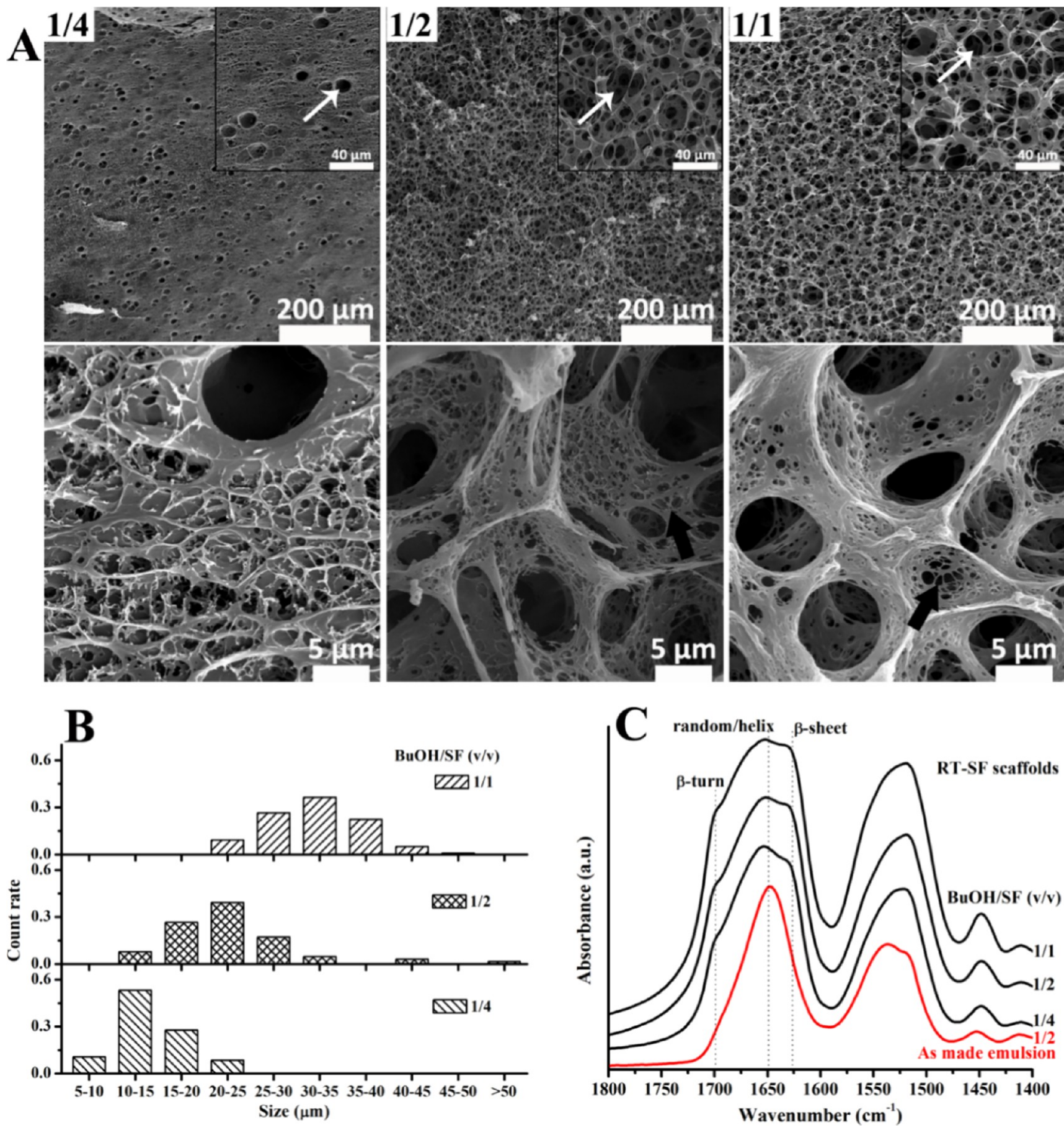

Figure 2. (A) SEM images and (B) pore size analysis of RT-SF scaffolds formed from $\mathrm{O} / \mathrm{W}$ emulsions after $6 \mathrm{~h}$ at room temperature with BuOH/SF of $1 / 4,1 / 2$, and $1 / 1$ (macropores are marked by white arrows, and the nanofibrous SF matrix is marked by black arrows). (C) Fourier transform infrared (FT-IR) spectra of SF in the as-made $\mathrm{O} / \mathrm{W}$ emulsion with $\mathrm{BuOH} / \mathrm{SF}$ of $1 / 2$ and in the scaffolds formed from these emulsions after $6 \mathrm{~h}$ at room temperature.

arrangement and self-assembly of SF with the help of 1-butanol both at the interface and in the bulk aqueous solution.

The $\mathrm{O} / \mathrm{W}$ emulsions formed with various volume ratios of 1 butanol/SF aqueous solution (denoted $\mathrm{BuOH} / \mathrm{SF}$ throughout the text) were studied with an optical microscope (OM). When $\mathrm{BuOH} / \mathrm{SF}$ increased from $1 / 4$ to $1 / 1$, the number of dispersed emulsion droplets increased (Figure 1A), whereas the average droplet size only slightly increased from $6.6 \pm 6.0$ to $11.9 \pm 3.6$ $\mu \mathrm{m}$ without any significant difference $(P>0.05$, Figure $1 \mathrm{C})$; this is because under the same experimental conditions the physical nature of the two phases and SF acting as the emulsifier jointly determined the interfacial characteristics of the $\mathrm{O} / \mathrm{W}$ emulsions; ${ }^{33}$ for $\mathrm{BuOH} / \mathrm{SF}$ of $1 / 1$, spherical emulsion droplets packed densely and squeezed each other, resulting in slight deformation at the edge (Figure 1A, marked by arrows). Statistical analysis on emulsion droplet size showed a Gaussian distribution for the three emulsions (Figure 1B). As a comparison, without the presence of SF, stirring the mixture of 1-butanol/water could not form an emulsion, confirming SF's vital role in the preparation and stabilization of the $\mathrm{O} / \mathrm{W}$ 

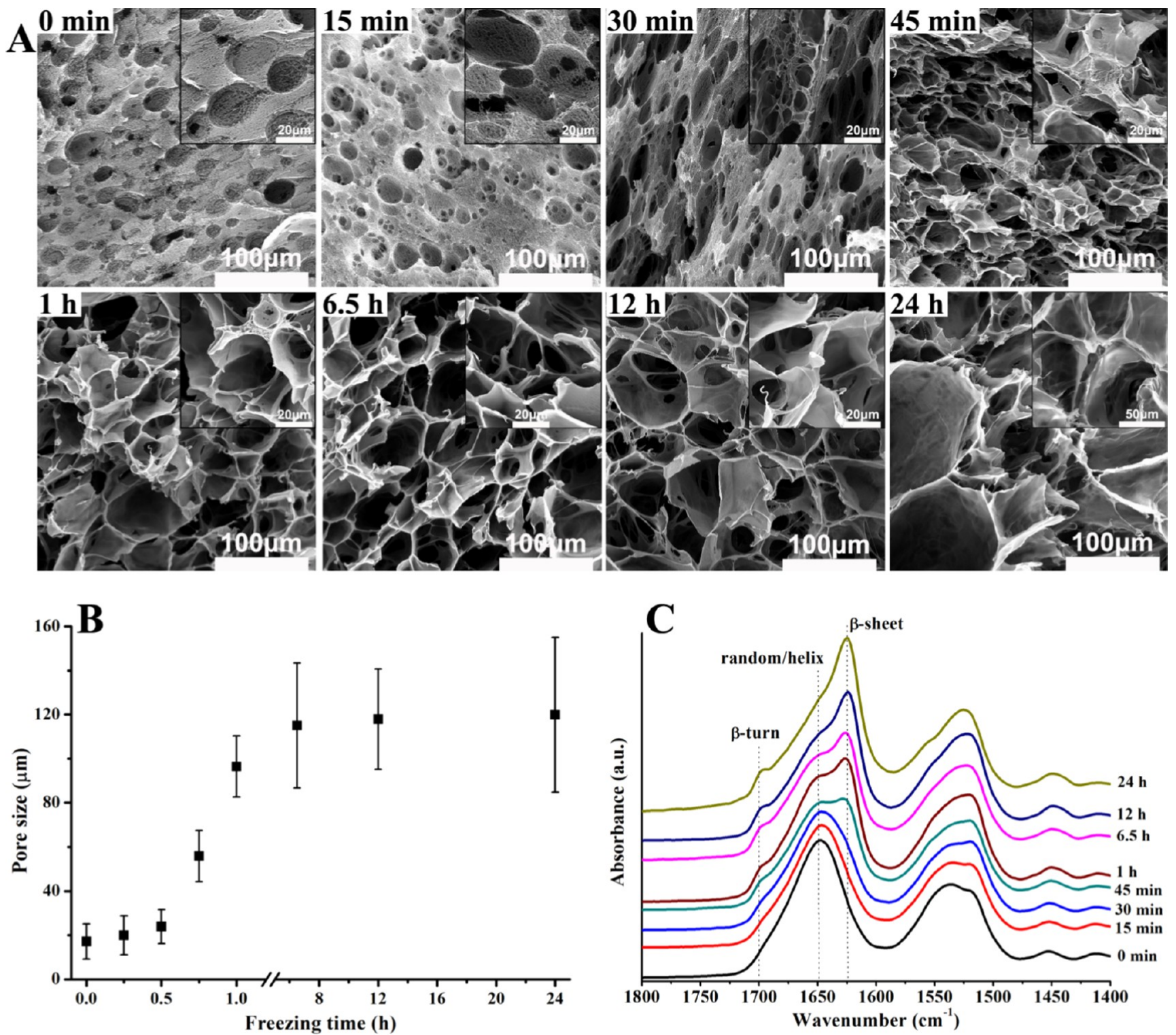

Figure 3. Real-time (A) SEM investigation, (B) pore size analysis, and (C) FT-IR spectra of SF through the evolution from O/W emulsion (BuOH/ $\mathrm{SF}$ of $1 / 2$ ) to scaffold formation under freezing at $-20{ }^{\circ} \mathrm{C}$ for different periods.

emulsions because no other surface-active agent was added. SF adsorbed at the interface not only decreased the interfacial tension but also acted as a protective barrier to prevent coalescence of the emulsion droplets upon contact with each other and/or Ostwald ripening. Moreover, SF in the continuous aqueous solution increased the viscosity of the system, thus reducing the frequency of droplet encountering. All of these factors contributed to stabilization of SF selfemulsified emulsions. ${ }^{34}$

The O/W emulsions were stable until solidification into RT$\mathrm{SF}$ scaffolds after $6 \mathrm{~h}$ at room temperature because neither emulsion breaking nor flocculation (creaming and/or sedimentation) was observed. Scanning electron microscopy (SEM) study of RT-SF scaffolds clearly shows that the macropores (Figure 2A, white arrows) were derived from the emulsion droplets that remained dispersed in the solidified SF matrix because they showed similar trends with the emulsion droplets as observed in the $\mathrm{O} / \mathrm{W}$ emulsions (Figure 1A,B) in terms of pore quantities and pore size distributions regarding variation of $\mathrm{BuOH} / \mathrm{SF}$ (Figure $2 \mathrm{~A}, \mathrm{~B}$ ). The average pore size increased from $14.2 \pm 3.6$ to $31.9 \pm 5.2 \mu \mathrm{m}$ when $\mathrm{BuOH} / \mathrm{SF}$ increased from $1 / 4$ to $1 / 1$, which was just slightly bigger than the droplet size of the corresponding emulsions (Figure 1C), suggesting excellent stability of the $\mathrm{O} / \mathrm{W}$ emulsions over time until solidification of SF. These findings confirm that SF is a superior emulsifier.

FT-IR spectra of SF in the emulsions and in the RT-SF scaffolds are shown in Figure 2C. For SF in the freshly made emulsion, the characteristic broad peak at $1650 \mathrm{~cm}^{-1}$ was assigned to the random coil/helical conformation; in addition, a tiny convex peak at around $1700 \mathrm{~cm}^{-1}$, which was related to the $\beta$-turn conformation, ${ }^{35}$ could be observed, suggesting that upon mixing 1-butanol with SF solution to form the $\mathrm{O} / \mathrm{W}$ emulsion conformational transition of SF to $\beta$-turn first occurred, which was consistent with the report on 1-butanol-induced conformational transition of the SF film. ${ }^{36}$ This is because 1-butanol has weak ability to induce conformational transition of $\mathrm{SF}^{36}$ and $\beta$ turn, which is usually associated with the formation of $\beta$-sheet and only involves local arrangement of four residues in SF chains, is easier to achieve than $\beta$-sheet. ${ }^{35}$ For SF in the RT-SF 
scaffolds, in addition to the dominant broad peak at $1650 \mathrm{~cm}^{-1}$, shoulder peaks at 1700 and $1625 \mathrm{~cm}^{-1}$, which were attributable to $\beta$-turn and $\beta$-sheet structures, respectively, ${ }^{35}$ could be observed, indicating that 1 -butanol induced conformational transition to $\beta$-sheet, which acted as physical cross-linkers, resulting in solidification of the $\mathrm{O} / \mathrm{W}$ emulsions to form $\mathrm{RT}$-SF scaffolds. With an increase in $\mathrm{BuOH} / \mathrm{SF}$ from $1 / 4$ to $1 / 1$, the $\beta$-sheet content increased, indicated by the slightly increasing peak at $1625 \mathrm{~cm}^{-1}$.

Interestingly, RT-SF scaffolds solidified from dense emulsions (e.g., $\mathrm{BuOH} / \mathrm{SF}$ of $1 / 1$ ) showed a hierarchically porous structure, featuring emulsion-templated macropores, interconnected micropores, and nanofibrous SF matrix (Figure 2A, black arrows). The interconnected porous structure usually forms after solidification of the continuous phase in a dense emulsion where dispersed droplets squeezed each other, e.g., high internal phase emulsion-templated porous materials, ${ }^{37}$ whereas the SF matrix is similar to the nanofibrous structure in ethanol-induced SF gels. ${ }^{38}$ Although SF materials with such hierarchically porous structures are highly desirable, all RT-SF scaffolds were friable and easily broken up upon handling, which restricted their applications.

Mechanism of Scaffold Formation through Freezing the O/W Emulsion. Although RT-SF scaffolds were mechanically friable, we found that by simply freezing these $\mathrm{O} / \mathrm{W}$ emulsions at $-20{ }^{\circ} \mathrm{C}$ overnight, robust SF scaffolds (F-SF scaffolds; Figure S2, see Supporting Information) were obtained without any post-treatment. ${ }^{29}$ To further clarify the scaffold formation mechanism of this facile technique, the evolution from the $\mathrm{O} / \mathrm{W}$ emulsion to porous scaffold formation under freezing was tracked with SEM and FT-IR.

As shown in Figure 3A, the freshly prepared emulsion showed a morphology corresponding well to the OM observations (Figure 1A), i.e., pores with sizes ranging from 5 to $30 \mu \mathrm{m}$ were derived from the dispersed droplets, whereas the nanofibrous network in the surrounding matrix maintained the morphology of SF in the continuous aqueous solution. After 15 min of freezing at $-20{ }^{\circ} \mathrm{C}$, morphology barely changed compared to that of the freshly prepared emulsion except that a few small pores of around $5 \mu \mathrm{m}$ appeared in the nanofibrous network, which could be vacancies left by ice crystals frozen from the aqueous phase. When the freezing time increased to $30 \mathrm{~min}$, although emulsion droplet-templated pores did not change, ice-templated pores in the nanofibrous network increased in terms of both quantity and size and a few smooth and dense SF membranes appeared as pore walls. This should be because ice nuclei increased in number and began to grow.

As freezing continued to $1 \mathrm{~h}$, the morphology changed dramatically because of the rapid growth of ice crystals, average pore size rapidly increased to around $100 \mu \mathrm{m}$ (Figure 3B), and more smooth SF membranes formed as pore walls, whereas the nanofibrous network reduced. Thus, it became impossible to distinguish 1-butanol droplet-templated pores from icetemplated pores. After $6.5 \mathrm{~h}$ of freezing, no nanofibrous network could be found and the homogeneous porous scaffold showed only the smooth and dense SF membrane pore walls, whereas the average pore size increased slightly to around 110 $\mu \mathrm{m}$ (Figure $3 \mathrm{~B}$ ), suggesting that growth of ice crystals was restricted after $1 \mathrm{~h}$ of freezing because the dense SF membrane pore walls could hinder ice growth. A further increase in freezing time did not alter the morphology and average pore size (Figure 3B), implying that ice growth completed. Moreover, pore size analysis shows that emulsion-templated pores increased from $5-30 \mu \mathrm{m}$ to around $110 \mu \mathrm{m}$ (Figure 3B), suggesting that when ice growth pushed emulsion droplets to move close to each other under freezing at $-20{ }^{\circ} \mathrm{C}$ adequate stability of the $\mathrm{O} / \mathrm{W}$ emulsion was maintained because coalescence occurred to a limited extent.

Real-time FT-IR studies are shown in Figure 3C. As mentioned above, 1-butanol-induced conformational transition of SF to $\beta$-turn first occurred in the freshly made $\mathrm{O} / \mathrm{W}$ emulsion and with the increase in freezing time to $24 \mathrm{~h}$ the convex peak at around $1700 \mathrm{~cm}^{-1}$ gradually increased to become a small peak. On the other hand, after $30 \mathrm{~min}$ of freezing, a shoulder at $1625 \mathrm{~cm}^{-1}$ appeared, indicating that conformational transition to $\beta$-sheet via intermediate of $\beta$-turn took place. ${ }^{35}$ As freezing continued to $1 \mathrm{~h}$, the shoulder at 1625 $\mathrm{cm}^{-1}$ increased significantly to become a peak that surpassed the peak at $1650 \mathrm{~cm}^{-1}$, suggesting fast conformational transition from random coil/helical to $\beta$-sheet. Apparently, this was caused by formation and rapid growth of ice crystals as observed with SEM (Figure 3A), which not only resulted in concentration of SF aqueous solution, leading to a shorter chain-chain distance that would facilitate 1-butanol-induced self-assembly of SF, but also pushed these SF assemblies and molecules moving toward each other to aggregate into dense SF membranes as pore walls. As controls, SF solution and RTSF scaffolds were frozen at $-20{ }^{\circ} \mathrm{C}$ for $24 \mathrm{~h}$, respectively. After thawing, for the solution sample, only SF aqueous solution was recovered, indicating that shearing force generated by ice formation is inadequate to induce conformational transition of SF from water-soluble random coil/helical to water-insoluble $\beta$ sheet; ${ }^{39}$ for the scaffold sample, the resulting scaffolds were still mechanically friable and showed no difference in morphology (data not shown), suggesting that once conformational transition to $\beta$-sheet, acting as physical cross-linkers, has completed, ice formation is unable to change the structure. These results point to the importance of kinetic sequence or synchronism between 1-butanol-induced SF conformational transition and freezing-induced ice formation for the present technique. That is, ice formation and growth could accelerate and enhance the conformational transition of SF to $\beta$-sheet only after the dehydration activity of 1-butanol has initiated the transition and before the transition is complete, as presented here. Therefore, compared to that for RT-SF scaffolds (Figure $2 \mathrm{C})$, the $\beta$-sheet content was much higher for F-SF scaffolds. As freezing continued to $24 \mathrm{~h}$, the peak at $1625 \mathrm{~cm}^{-1} \mathrm{kept}$ increasing, whereas the peak at $1650 \mathrm{~cm}^{-1}$ decreased to become a shoulder, suggesting that SF molecules could still move to accomplish conformational transition at $-20{ }^{\circ} \mathrm{C}$, although their movement would indeed slow down, as dictated from the energy perspective. Meanwhile, SEM study shows that the morphology and average pore size were essentially unchanged after $1 \mathrm{~h}$ of freezing (Figure 3A,B). Therefore, F-SF scaffolds with a similar porous structure yet different $\beta$-sheet content (determining adjustable degradation behavior) could be obtained using this technique by simply controlling the freezing periods.

Apparently, freezing-caused structural change in the resulting F-SF scaffolds, as indicated by SEM and FT-IR studies, is the reason for the improved mechanical properties, compared to those of the friable RT-SF scaffolds, which is most likely due to the formation of large $\beta$-sheet domains, same as reported for ethanol-induced weak SF gels. ${ }^{40}$ Ice formation from the SF solution under freezing not only resulted in a higher $\beta$-sheet content and relatively tight structure (indicated by the smooth 


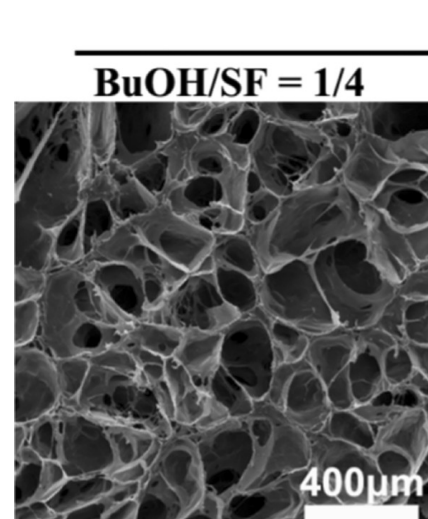

\section{$7 \mathrm{wt} \%$ SF solution}
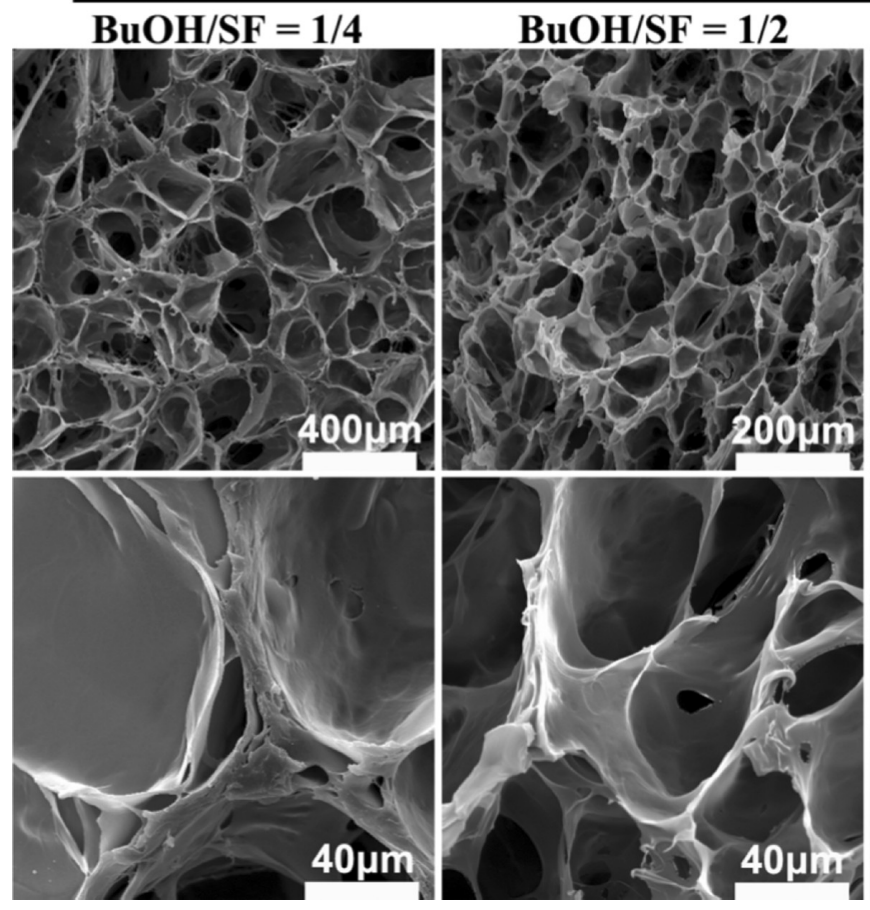

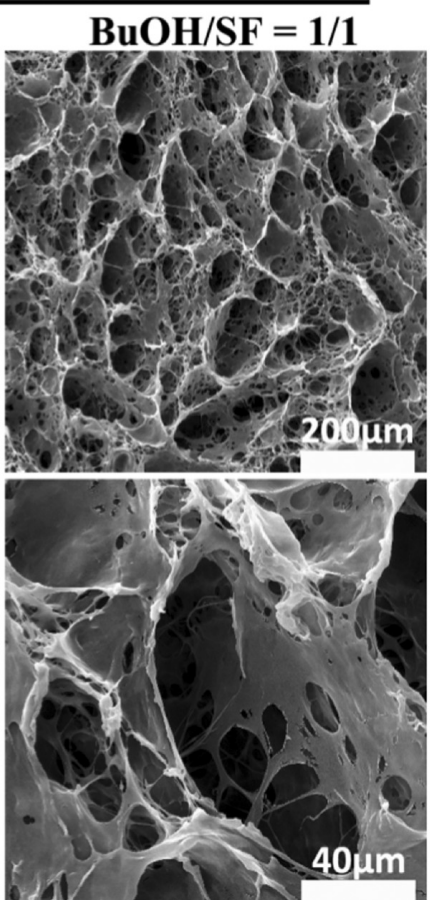

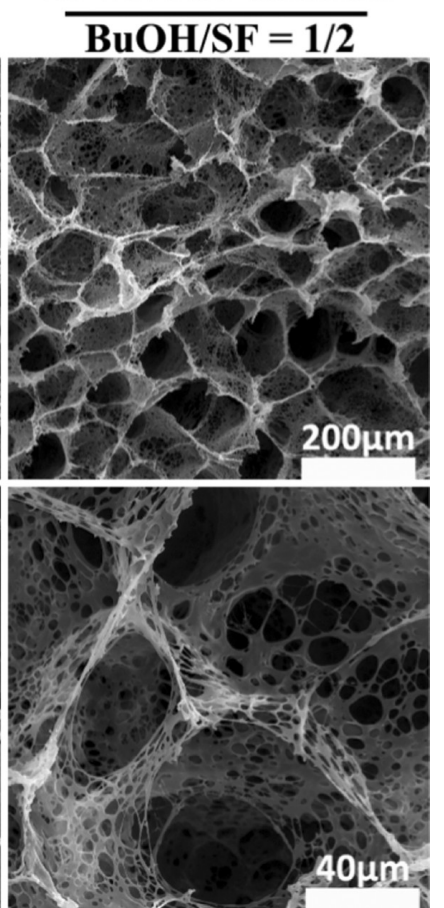

Figure 4. SEM images of F-SF scaffolds prepared from O/W emulsions with different $\mathrm{BuOH} / \mathrm{SF}$ after freezing at $-20{ }^{\circ} \mathrm{C}$ for $24 \mathrm{~h}$.

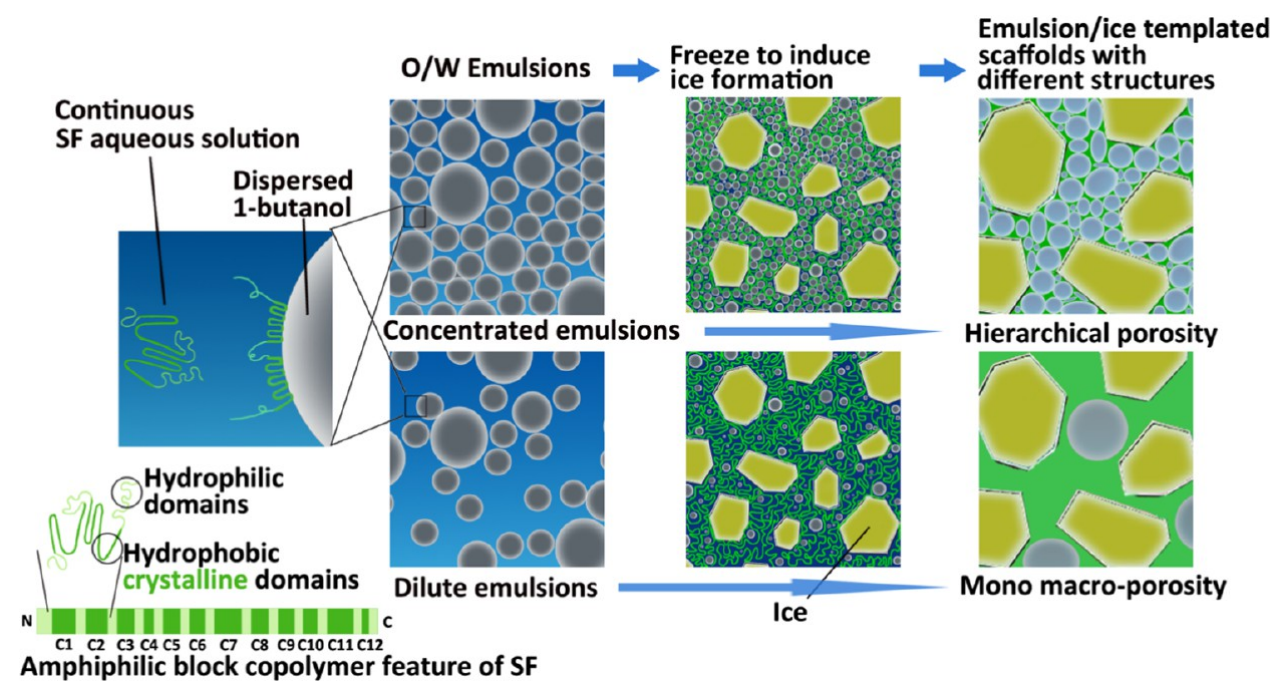

Figure 5. Schematic illustration of the SF self-emulsified O/W emulsion-ice dual template mechanism for the preparation of 3D porous SF scaffolds with different structures.

and dense membrane pore walls) but also might restrict the growth of $\beta$-sheet domains and thereby might form numerous small-sized $\beta$-sheet domains. These structural features can disperse stress effectively and thus improve the mechanical properties. $^{40-42}$ Moreover, volume expansion during ice formation would generate a strong shearing force, ${ }^{39}$ which might biaxially stretch SF assemblies/molecules to achieve a certain extent of molecular orientation in the pore walls, the effect of which resembles the blow molding process in plastic industry. ${ }^{43}$ We have previously reported that uniaxial extension of SF film indeed enhanced its toughness and strength. ${ }^{44}$

Fabrication of F-SF Scaffolds with a Controllable Hierarchically Porous Structure. The effects of $\mathrm{BuOH} / \mathrm{SF}$ on the structure of F-SF scaffolds were characterized with SEM.
For $\mathrm{BuOH} / \mathrm{SF}$ of $1 / 4$ and $1 / 2$, the resulting scaffolds showed a homogenous porous structure with open mono macropores as well as smooth and dense membrane pore walls. When $\mathrm{BuOH} /$ $\mathrm{SF}$ increased to $1 / 1$, the resulting scaffold showed a hierarchically porous structure featuring open macropores, interconnected micropores, and micro-/nanofibrous structures in the pore walls (Figure 4).

On the basis of above investigations on the stability of $\mathrm{O} / \mathrm{W}$ emulsions at room temperature or $-20^{\circ} \mathrm{C}$, a SF self-emulsified $\mathrm{O} / \mathrm{W}$ emulsion-ice dual template mechanism is proposed for the preparation of robust F-SF scaffolds with controllable hierarchical porosities, as depicted in Figure 5. When 1-butanol is emulsified in $\mathrm{SF}$ aqueous solution with stirring to form $\mathrm{O} / \mathrm{W}$ emulsions, SF molecules, acting as the self-emulsifier, effectively 
Table 1. Selected Physical Properties of F-SF Scaffolds Prepared by Freezing the O/W Emulsions at $-20{ }^{\circ} \mathrm{C}$ for $24 \mathrm{~h}$ with Different $\mathrm{BuOH} / \mathrm{SF}^{a}$

\begin{tabular}{lccc}
\multicolumn{1}{c}{$\mathrm{BuOH} / \mathrm{SF}(\mathrm{v} / \mathrm{v})$} & $1 / 4$ & $1 / 2$ & $1 / 1$ \\
pore size $(\mu \mathrm{m})$ & $232 \pm 55$ & $130 \pm 37$ & $114 \pm 21$ \\
porosity $(\%)$ & $95.0 \pm 0.1$ & $95.7 \pm 0.1$ & $95.0 \pm 0.2$ \\
water uptake $(\mathrm{g} / \mathrm{g})$ & $19.4 \pm 0.3$ & $21.3 \pm 1.0$ & $17.7 \pm 0.7$ \\
water content $(\%)$ & $95.1 \pm 0.1$ & $95.5 \pm 0.2$ & $94.6 \pm 0.2$ \\
apparent density $\left(\mathrm{mg} / \mathrm{cm}^{3}\right)$ & $67.5 \pm 1.4$ & $57.6 \pm 0.7$ & $68.0 \pm 2.8$ \\
compressive modulus $(\mathrm{kPa})$ & $364 \pm 111$ & $228 \pm 76$ & $13 \pm 3.82$ \\
compressive strength $(\mathrm{kPa})$ & $23 \pm 7$ & $4.0 \pm 1.3$ & $3.8 \pm 1.2$ \\
specific compressive modulus $\left(\mathrm{MPa} \cdot \mathrm{cm}^{3} / \mathrm{g}\right)$ & $5.4 \pm 1.6$ & $234 \pm 45$ & $230 \pm 85$ \\
specific compressive strength $\left(\mathrm{kPa} \cdot \mathrm{cm}^{3} / \mathrm{g}\right)$ & $338 \pm 98$ & & \\
\hline
\end{tabular}

stabilize the emulsions. During freezing at $-20^{\circ} \mathrm{C}$, apart from the emulsion template, ice forms from the SF solution to serve as the second pore template. In addition, ice formation not only enhances the conformational transition of SF to $\beta$-sheet, which is initiated by 1-butanol to act as physical cross-linkers, but also improves structural performance that leads to the formation of robust porous F-SF scaffolds without the need for any further post-treatment. Moreover, by tuning the emulsion droplet volume fraction, F-SF scaffolds with controlled hierarchical porosities can be prepared. For a diluted emulsion $(\mathrm{BuOH} / \mathrm{SF}$ of $1 / 4$ and $1 / 2$ ), droplets are dispersed with enough distance from each other that the resulting F-SF scaffolds show a mono macroporous structure with smooth and dense membrane pore walls templated by ice crystals and partially coalesced droplets. For a concentrated emulsion $(\mathrm{BuOH} / \mathrm{SF}$ of $1 / 1)$, droplets pack densely, leading to interconnected micropores and micro-/ nanofibrous structures in the pore walls, which, together with the macropores templated by ice crystals and partially coalesced droplets, demonstrate a hierarchically porous structure of the resulting F-SF scaffolds (Figure 4).

It is well known that the increasing viscosity of the continuous phase can impart added stability to the emulsion. ${ }^{34}$ With this method to enhance emulsion stability in our system, coalescence of the 1-butanol droplets may be reduced or avoided, which assures sufficient droplet quantity and interface area. Therefore, when ice formation pushes emulsion droplets to move close to each other under freezing at $-20{ }^{\circ} \mathrm{C}$, the dispersed droplets can realize a dense packing state with a reduced $\mathrm{BuOH} / \mathrm{SF}$ compared to $\mathrm{BuOH} / \mathrm{SF}$ of $1 / 1$ as described above, thus also leading to the formation of a hierarchically porous structure. To verify this hypothesis, we used $13 \mathrm{wt} \% \mathrm{SF}$ solution, which had a higher viscosity than that of 7 wt \% solution, ${ }^{45}$ as the aqueous phase to prepare the $\mathrm{O} / \mathrm{W}$ emulsion at a reduced $\mathrm{BuOH} / \mathrm{SF}$ of $1 / 2$. After freezing at $-20{ }^{\circ} \mathrm{C}$ for 24 $\mathrm{h}$, the resulting scaffolds indeed showed a hierarchically porous structure featuring open macropores templated by ice crystals, interconnected micropores, and micro-/nanofibrous structures in the pore walls templated by the densely packed droplets (Figure 4).

Physical Properties of F-SF Scaffolds. Apart from controlling the hierarchical porosities of F-SF scaffolds, the effects of $\mathrm{BuOH} / \mathrm{SF}$ on the pore size, porosity, water uptake, and mechanical properties of F-SF scaffolds were tested.

With an increase in $\mathrm{BuOH} / \mathrm{SF}$ from $1 / 4$ to $1 / 1$, the average pore size of F-SF scaffolds decreased from 230 to $110 \mu \mathrm{m}$, whereas porosities were essentially unchanged with values of about 95\% (Table 1). The same low SF content (7 wt \%) and the emulsion-ice dual templates jointly contributed to the similar high porosities of F-SF scaffolds, although $\mathrm{BuOH} / \mathrm{SF}$ could control the hierarchical structure of their pores (Figure 4). Moreover, hexane, used in the test of porosities via the liquid displacement method, could neither swell nor shrink the SF matrix; ${ }^{46}$ therefore, the high porosities suggest that the pores of F-SF scaffolds are interconnected, which can provide abundant $3 \mathrm{D}$ space for cell growth and help mass exchange to transport nutrition in and metabolite out in tissue engineering. ${ }^{4}$

Scaffolds for tissue engineering are required to have strong ability for water uptake, which favors cell adhesion and growth. $^{47,48}$ When $\mathrm{BuOH} / \mathrm{SF}$ increased from $1 / 4$ to $1 / 1$, water uptakes of F-SF scaffolds were in the range of $18-21 \mathrm{~g} / \mathrm{g}$, which correlated to water contents of about $95 \%$ for the wet FSF scaffolds (Table 1). The high water uptakes could be due to the hydrophilic nature of SF and the interconnected high porosities of F-SF scaffolds. ${ }^{48}$

Scaffolds for tissue engineering are placed with stringent mechanical requirements; they must not only support cell activities and new tissue integration both in vitro and in vivo but also resist handling during surgery and withstand postimplantation hydrodynamic and mechanical stresses and strains in vivo. ${ }^{49}$ Compression curves of F-SF scaffolds in wet conditions were recorded, and the corresponding compressive modulus and strength are listed in Table 1 . With the increase of $\mathrm{BuOH} / \mathrm{SF}$ from $1 / 4$ to $1 / 1$, the compressive modulus first deceased from 360 to $230 \mathrm{kPa}$ and then increased slightly to $260 \mathrm{kPa}$; the compressive strength showed a similar trend. According to the theoretical framework established for mechanical behavior of low-density porous scaffolds for tissue engineering, the compressive modulus and strength of the porous scaffolds are proportional to the square of the relative density (ratio of the apparent density including the pores and the density of the material forming the scaffold) and also proportional to the modulus of the material forming the scaffold. $^{49-51}$ Therefore, to eliminate the influence due to different porosities, the specific compressive modulus and strength were calculated by normalizing the compressive modulus and strength by the scaffold density (Table 1 ). When $\mathrm{BuOH} / \mathrm{SF}$ increased from $1 / 4$ to $1 / 1$, the specific compressive modulus of F-SF scaffolds decreased from 5400 to $3800 \mathrm{kPa} \cdot \mathrm{cm}^{3} / \mathrm{g}$. These values were an order of magnitude higher than those of SF scaffolds prepared by salt-leaching with a methanol post-treatment (about $200 \mathrm{kPa} \cdot \mathrm{cm}^{3} / \mathrm{g}$ ) and comparable to the value of SF composite scaffolds reinforced with $200 \%$ silk particles (ranging from 4600 to $7700 \mathrm{kPa} \cdot \mathrm{cm}^{3}$ / g). ${ }^{49}$ Theoretically, pore wall or local strut characteristics determine the global mechanical properties of porous scaffolds. ${ }^{49,50}$ For SF-based materials, the mechanical properties 


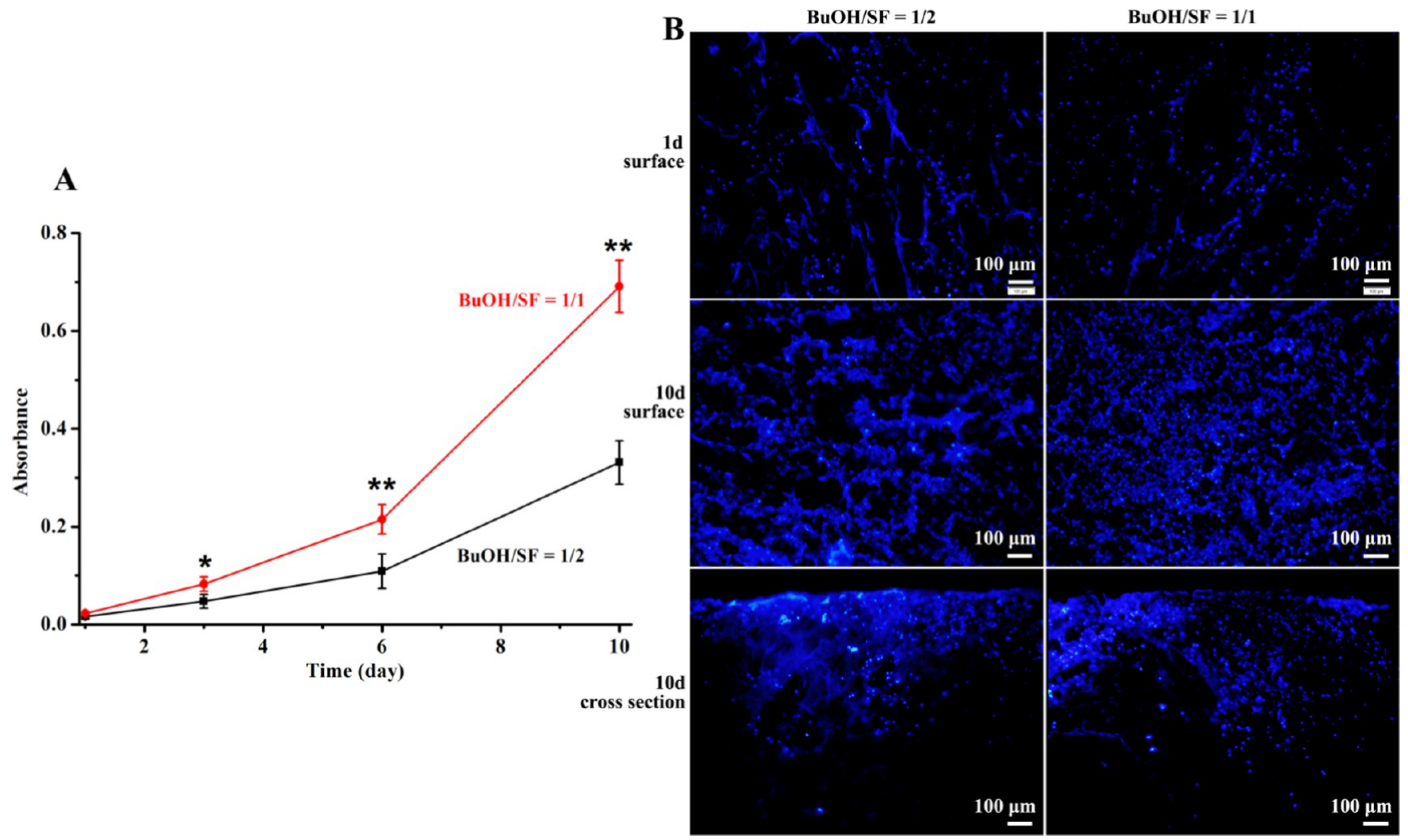

Figure 6. (A) MTT assay for the proliferation of L929 fibroblasts cultured on F-SF scaffolds prepared with BuOH/SF of 1/2 (mono macroporosity) and 1/1 (hierarchical porosity); * and ** denote $P<0.05$ and $P<0.01$, respectively; $N=4$. (B) Fluorescence microscopy images of L929 fibroblasts cultured on F-SF scaffolds. Cells were stained with (4',6-diamidino-2-phenylindole) for nuclei after culture time of 1 day and 10 days.

are closely linked to the secondary structure and even higher level of aggregation structure of SF, which are not merely determined by the primary amino acid sequence but more depend on the control by the processing techniques employed. ${ }^{52}$ As described above, the significantly improved mechanical properties of F-SF scaffolds were due to the enhanced toughness and strength of pore walls caused by the freezing-induced structural change.

Bioactivity of SF Scaffolds. To assess the effects of different hierarchical porosities of F-SF scaffolds on their bioactivity toward L929 murine fibroblast, as a representative mammalian cell, the 3-(4,5-dimethylthiazol-2-yl)-2,5-diphenyltetrazolium bromide (MTT) assay was employed to evaluate the viability and proliferation of L929 cells cultured on F-SF scaffolds for 10 days. F-SF scaffolds prepared with $\mathrm{BuOH} / \mathrm{SF}$ of $1 / 2$ and $1 / 1$ were chosen because they shared similar pore size, porosity, and mechanical properties (Table 1) whereas different hierarchical porosities (Figure 4). After seeding with the same amount of cells on each scaffold $\left(1 \times 10^{4}\right.$ cells $\left./ \mathrm{cm}^{2}\right)$ and incubating for 1 day, we did not notice any cells grew on the culture plates, neither did we find any dead cells on the scaffolds with live/dead staining assays, indicating that all of the seeded cells adhered on the scaffolds; when the culture time extended through 10 days, L929 cells could further grow and proliferate on both scaffolds (Figure 6), suggesting no cytotoxicity of F-SF scaffolds prepared through our strategy. Moreover, after 3 days of culture, cells on F-SF scaffolds with hierarchical porosity $(\mathrm{BuOH} / \mathrm{SF}$ of $1 / 1)$ were significantly more than cells on F-SF scaffolds with mono macroporosity $(\mathrm{BuOH} / \mathrm{SF}$ of $1 / 2)(P<0.05)$; with culture time increased to 6 and 10 days, the discrepancy between them became even more significant $(P<0.01$, Figure $6 \mathrm{~A})$. The enhancement of cellular activity by the hierarchically porous structure was also demonstrated by the fluorescence microscopy images showing higher degree of cell spreading across the scaffolds (both on the surface or into the scaffold) after 10 days of culture compared to that across F-SF scaffolds with mono macroporosity (Figure $6 \mathrm{~B})$. These results clearly indicate the crucial role of the micropores and micro-/nanofibrous structures in the walls of macropores, which mimic extracellular matrix to provide an optimal environment for cell adhesion, migration, and proliferation through the scaffolds. ${ }^{5,53}$ The findings presented here point to great potentials of F-SF scaffolds with such a hierarchically porous structure for a broad range of tissue engineering and other related biomedical applications.

\section{CONCLUSIONS}

In the present study, SF was used as the self-emulsifier to prepare $\mathrm{O} / \mathrm{W}$ emulsions by taking advantage of SF's strong surface activity using 1-butanol as the oil phase and SF aqueous solution as the water phase without addition of any other surface-active agent. Thereafter, 3D porous F-SF scaffolds with high porosity and strong mechanical properties were prepared through emulsion-ice dual templates without the need for any further post-treatment due to 1-butanol-induced conformational transition of SF to $\beta$-sheet, which acted as the crosslinkers. It was found that the freezing-caused structural change was the reason for the improved mechanical properties of the resulting F-SF scaffolds, and by tuning the dispersed droplet volume fraction, F-SF scaffolds with controllable hierarchically porous structures were achieved. Therefore, by ingeniously taking SF as both the emulsifier and the matrix material, we 
provide a simple strategy to preparing SF scaffolds yet with versatile control on both the gross shape and the microscale hierarchically porous structure. Furthermore, it was found that F-SF scaffolds with hierarchical porosity showed significantly higher bioactivity toward L929 fibroblasts than that of F-SF scaffolds with mono macroporosity, highlighting the great asset of hierarchically porous F-SF scaffolds prepared using this strategy for the broad applications in tissue engineering and other related biomedical areas. Moreover, the strong surfaceactive characteristic of SF presented here, in addition to its distinct advantages, sheds a bright light on the application of SF in the vast range of emulsion technologies, especially in cosmetic-, food-, and biomedical-related areas.

\section{EXPERIMENTAL SECTION}

SF Self-Emulsified O/W Emulsions. SF aqueous solution was prepared following well-established protocols. ${ }^{20}$ A 7 wt \% SF solution was used in this study unless otherwise specified. 1Butanol was added into SF aqueous solution at various volume ratios under stirring (400 $\mathrm{rpm}$ ) for $2 \mathrm{~min}$ at room temperature to form $\mathrm{O} / \mathrm{W}$ emulsions. The obtained emulsions were kept at room temperature for a series of periods until solidification to form RT-SF scaffolds. At each time point, these emulsions were observed under an optical microscope (OM, Olympus BX-51, Japan), the emulsion droplet size and its distribution were analyzed with Atlas software (Tescan, Czech), and more than 100 droplets were randomly selected for the statistical analysis. RT-SF scaffolds were studied with SEM and FT-IR.

Fabrication of Porous SF Scaffolds. Freshly prepared O/ $\mathrm{W}$ emulsions were casted into a custom-built mold and frozen at $-20{ }^{\circ} \mathrm{C}$ for $24 \mathrm{~h}$ to fabricate porous F-SF scaffolds following our established procedure. ${ }^{29}$ After thawing and washing with deionized water to thoroughly remove 1-butanol, wet F-SF scaffolds (filled with water) with gross shape of the mold were obtained (Figure S2, see Supporting Information). Dry F-SF scaffolds could be obtained when needed through a further freeze-drying process.

To track the evolution from the $\mathrm{O} / \mathrm{W}$ emulsion to porous scaffold formation, the freshly prepared $\mathrm{O} / \mathrm{W}$ emulsions $(\mathrm{BuOH} / \mathrm{SF}$ of $1 / 2)$ were kept at $-20{ }^{\circ} \mathrm{C}$ for a series of periods; three samples were then taken out and immediately immersed into liquid nitrogen $\left(-196{ }^{\circ} \mathrm{C}\right)$, followed by lyophilization immediately. With such an ultrarapid cooling rate, both solute (SF) and solvent (water and 1-butanol) molecules were promptly immobilized (or vitrified) in situ, where amorphous ice formed instead of ice crystal, thus avoiding the structure and volume change due to ice crystal growth. $^{54}$ Therefore, with this approach, real-time investigation of emulsion (or scaffold) morphology and SF conformation during freezing the emulsions could be realized with SEM and FT-IR, respectively.

Characterizations. Test methods for SEM, FT-IR, porosity, apparent density, water uptake, mechanical properties, and cell culture of F-SF scaffolds are described in the Supporting Information.

\section{ASSOCIATED CONTENT}

\section{S Supporting Information}

The Supporting Information is available free of charge on the ACS Publications website at DOI: 10.1021/acsomega.7b01874.
Detailed characterization methods, photographs of mixtures of 1-butanol/SF aqueous solution, and F-SF scaffolds with various gross shapes are provided (PDF)

\section{AUTHOR INFORMATION}

\section{Corresponding Author}

*E-mail: zzshao@fudan.edu.cn.

ORCID

Xin Chen: 0000-0001-7706-4166

Zhengzhong Shao: 0000-0001-5334-4008

\section{Notes}

The authors declare no competing financial interest.

\section{ACKNOWLEDGMENTS}

This work is supported by the National Natural Science Foundation of China (Nos. 21574024, 21574023). The authors sincerely thank Prof. Yuyu Sun (University of Massachusetts Lowell) for his valuable suggestions.

\section{REFERENCES}

(1) Langer, R; Vacanti, J. P. Tissue Engineering. Science 1993, 260, 920-926.

(2) Zhang, L.; Webster, T. J. Nanotechnology and nanomaterials: Promises for improved tissue regeneration. Nano Today 2009, 4, 6680.

(3) Freed, L. E.; Engelmayr, G. C.; Borenstein, J. T.; Moutos, F. T.; Guilak, F. Advanced Material Strategies for Tissue Engineering Scaffolds. Adv. Mater. 2009, 21, 3410-3418.

(4) Hollister, S. J. Porous scaffold design for tissue engineering. Nat. Mater. 2005, 4, 518-524.

(5) Ng, R.; Zang, R.; Yang, K. K.; Liu, N.; Yang, S.-T. Threedimensional fibrous scaffolds with microstructures and nanotextures for tissue engineering. RSC Adv. 2012, 2, 10110-10124.

(6) Wei, G.; Ma, P. X. Partially nanofibrous architecture of 3D tissue engineering scaffolds. Biomaterials 2009, 30, 6426-6434.

(7) Cheng, K.; Kisaalita, W. S. Exploring cellular adhesion and differentiation in a micro-/nano-hybrid polymer scaffold. Biotechnol. Prog. 2010, 26, 838-846.

(8) Liu, X.; Smith, L. A.; Hu, J.; Ma, P. X. Biomimetic nanofibrous gelatin/apatite composite scaffolds for bone tissue engineering. Biomaterials 2009, 30, 2252-2258.

(9) Omenetto, F. G.; Kaplan, D. L. New Opportunities for an Ancient Material. Science 2010, 329, 528-531.

(10) Wen, J.; Yao, J.; Shao, Z. Recent Progress and Application of Non-bioactive Proteins in Material Fields. Acta Polym. Sin. 2011, 1223.

(11) Vepari, C.; Kaplan, D. L. Silk as a biomaterial. Prog. Polym. Sci. 2007, 32, 991-1007.

(12) Yan, J.; Zhou, G. Q.; Knight, D. P.; Shao, Z. Z.; Chen, X. WetSpinning of Regenerated Silk Fiber from Aqueous Silk Fibroin Solution: Discussion of Spinning Parameters. Biomacromolecules 2010, $11,1-5$.

(13) Wang, S.; Xu, T.; Yang, Y.; Shao, Z. Colloidal Stability of Silk Fibroin Nanoparticles Coated with Cationic Polymer for Effective Drug Delivery. ACS Appl. Mater. Interfaces 2015, 7, 21254-21262.

(14) Zhou, L.; Wang, Q.; Wen, J.; Chen, X.; Shao, Z. Preparation and characterization of transparent silk fibroin/cellulose blend films. Polymer 2013, 54, 5035-5042.

(15) Ni, Y.; Jiang, Y.; Wen, J.; Shao, Z.; Chen, X.; Sun, S.; Yu, H.; Li, $\mathrm{W}$. Construction of a functional silk-based biomaterial complex with immortalized chondrocytes in vivo. J. Biomed. Mater. Res., Part A 2014, 102, 1071-1078.

(16) Luo, K.; Shao, Z. A novel regenerated silk fibroin-based hydrogels with magnetic and catalytic activities. Chin. J. Polym. Sci. $2017,35,515-523$. 
(17) Tanaka, K.; Kajiyama, N.; Ishikura, K.; Waga, S.; Kikuchi, A.; Ohtomo, K.; Takagi, T.; Mizuno, S. Determination of the site of disulfide linkage between heavy and light chains of silk fibroin produced by Bombyx mori. Biochim. Biophys. Acta, Protein Struct. Mol. Enzymol. 1999, 1432, 92-103.

(18) Zhou, C.-Z.; Confalonieri, F.; Jacquet, M.; Perasso, R.; Li, Z.-G.; Janin, J. Silk fibroin: Structural implications of a remarkable amino acid sequence. Proteins 2001, 44, 119-122.

(19) Braun, F. N.; Viney, C. Modelling self assembly of natural silk solutions. Int. J. Biol. Macromol. 2003, 32, 59-65.

(20) Gong, Z.; Huang, L.; Yang, Y.; Chen, X.; Shao, Z. Two distinct $\beta$-sheet fibrils from silk protein. Chem. Commun. 2009, 7506-7508.

(21) Hao, R.; Zhang, J.; Xu, T.; Huang, L.; Yao, J.; Chen, X.; Shao, Z. Characterization and assembly investigation of a dodecapeptide hydrolyzed from the crystalline domain of Bombyx mori silk fibroin. Polym. Chem. 2013, 4, 3005-3011.

(22) Valluzzi, R.; Gido, S. P.; Zhang, W.; Muller, W. S.; Kaplan, D. L. Trigonal Crystal Structure of Bombyx mori Silk Incorporating a Threefold Helical Chain Conformation Found at the Air-Water Interface. Macromolecules 1996, 29, 8606-8614.

(23) Valluzzi, R.; Gido, S. P. The crystal structure of Bombyx mori silk fibroin at the air-water interface. Biopolymers 1997, 42, 705-717.

(24) Valluzzi, R.; Gido, S. P.; Muller, W.; Kaplan, D. L. Orientation of silk III at the air-water interface. Int. J. Biol. Macromol. 1999, 24, 237242.

(25) Yang, Y.; Dicko, C.; Bain, C. D.; Gong, Z.; Jacobs, R. M. J.; Shao, Z.; Terry, A. E.; Vollrath, F. Behavior of silk protein at the airwater interface. Soft Matter 2012, 8, 9705-9712.

(26) Gebhardt, R; Vendrely, C.; Burghammer, M.; Riekel, C. Characterization of the Boundary Zone of a Cast Protein Drop: Fibroin $\beta$-Sheet and Nanofibril Formation. Langmuir 2009, 25, 63076311.

(27) Peng, W.; Wang, L.; Qiao, X.; Shao, Z.; Sun, K. Emulsification Activity of Natural Silk Fibroin. Chem. J. Chin. Univ. 2015, 36, 10121018.

(28) Bhattacharjee, P.; Kundu, B.; Naskar, D.; Kim, H.-W.; Maiti, T. K.; Bhattacharya, D.; Kundu, S. C. Silk scaffolds in bone tissue engineering: An overview. Acta Biomater. 2017, 63, 1-17.

(29) Cao, Z.; Wen, J.; Yao, J.; Chen, X.; Ni, Y.; Shao, Z. Facile fabrication of the porous three-dimensional regenerated silk fibroin scaffolds. Mater. Sci. Eng., C 2013, 33, 3522-3529.

(30) Stephenson, R.; Stuart, J. Mutual binary solubilities: wateralcohols and water-esters. J. Chem. Eng. Data 1986, 31, 56-70.

(31) Hermanson, K. D.; Huemmerich, D.; Scheibel, T.; Bausch, A. R. Engineered Microcapsules Fabricated from Reconstituted Spider Silk. Adv. Mater. 2007, 19, 1810-1815.

(32) Li, G.; Zhou, P.; Shao, Z.; Xie, X.; Chen, X.; Wang, H.; Chunyu, L.; Yu, T. The natural silk spinning process. Eur. J. Biochem. 2001, 268, 6600-6606.

(33) Myers, D. Liquid-Fluid Interfaces. In Surfaces, Interfaces, and Colloids, 2nd ed.; Myers, D., Ed.; John Wiley \& Sons, Inc.: New York, 2002; pp 140-178.

(34) Myers, D. Emulsions. In Surfaces, Interfaces, and Colloids, 2nd ed.; Myers, D., Ed.; John Wiley \& Sons, Inc.: New York, 2002; pp 253-294.

(35) Chen, X.; Knight, D. P.; Shao, Z. Z. $\beta$-turn formation during the conformation transition in silk fibroin. Soft Matter 2009, 5, 27772781.

(36) Nazarov, R.; Jin, H.-J.; Kaplan, D. L. Porous 3-D Scaffolds from Regenerated Silk Fibroin. Biomacromolecules 2004, 5, 718-726.

(37) Viswanathan, P.; Chirasatitsin, S.; Ngamkham, K.; Engler, A. J.; Battaglia, G. Cell Instructive Microporous Scaffolds through Interface Engineering. J. Am. Chem. Soc. 2012, 134, 20103-20109.

(38) Gong, Z.; Yang, Y.; Huang, L.; Chen, X.; Shao, Z. Formation kinetics and fractal characteristics of regenerated silk fibroin alcogel developed from nanofibrillar network. Soft Matter 2010, 6, 12171223.
(39) Li, M.; Lu, S.; Wu, Z.; Yan, H.; Mo, J.; Wang, L. Study on porous silk fibroin materials. I. Fine structure of freeze dried silk fibroin. J. Appl. Polym. Sci. 2001, 79, 2185-2191.

(40) Li, Z.; Zheng, Z.; Yang, Y.; Fang, G.; Yao, J.; Shao, Z.; Chen, X. Robust Protein Hydrogels from Silkworm Silk. ACS Sustainable Chem. Eng. 2016, 4, 1500-1506.

(41) Su, D.; Yao, M.; Liu, J.; Zhong, Y.; Chen, X.; Shao, Z. Enhancing Mechanical Properties of Silk Fibroin Hydrogel through Restricting the Growth of $\beta$-Sheet Domains. ACS Appl. Mater. Interfaces 2017, 9, 17489-17498.

(42) Luo, K.; Yang, Y.; Shao, Z. Physically Crosslinked Biocompatible Silk-Fibroin-Based Hydrogels with High Mechanical Performance. Adv. Funct. Mater. 2016, 26, 872-880.

(43) Pham, X. T.; Thibault, F.; Lim, L. T. Modeling and simulation of stretch blow molding of polyethylene terephthalate. Polym. Eng. Sci. 2004, 44, 1460-1472.

(44) Yin, J.; Chen, E.; Porter, D.; Shao, Z. Enhancing the Toughness of Regenerated Silk Fibroin Film through Uniaxial Extension. Biomacromolecules 2010, 11, 2890-2895.

(45) Mo, C.; Holland, C.; Porter, D.; Shao, Z. Z.; Vollrath, F. Concentration State Dependence of the Rheological and Structural Properties of Reconstituted Silk. Biomacromolecules 2009, 10, 27242728.

(46) Kim, U. J.; Park, J.; Kim, H. J.; Wada, M.; Kaplan, D. L. Threedimensional aqueous-derived biomaterial scaffolds from silk fibroin. Biomaterials 2005, 26, 2775-2785.

(47) Kirdponpattara, S.; Khamkeaw, A.; Sanchavanakit, N.; Pavasant, P.; Phisalaphong, M. Structural modification and characterization of bacterial cellulose-alginate composite scaffolds for tissue engineering. Carbohydr. Polym. 2015, 132, 146-155.

(48) Ruan, Y.; Lin, H.; Yao, J.; Chen, Z.; Shao, Z. Preparation of 3D Fibroin/Chitosan Blend Porous Scaffold for Tissue Engineering Via a Simplified Method. Macromol. Biosci. 2011, 11, 419-426.

(49) Rajkhowa, R.; Gil, E. S.; Kluge, J.; Numata, K.; Wang, L.; Wang, X.; Kaplan, D. L. Reinforcing Silk Scaffolds with Silk Particles. Macromol. Biosci. 2010, 10, 599-611.

(50) Gibson, L. J. Biomechanics of cellular solids. J. Biomech. 2005, 38, 377-399.

(51) Harley, B. A.; Leung, J. H.; Silva, E. C. C. M.; Gibson, L. J. Mechanical characterization of collagen-glycosaminoglycan scaffolds. Acta Biomater. 2007, 3, 463-474.

(52) Shao, Z.; Vollrath, F. Materials: Surprising strength of silkworm silk. Nature 2002, 418, 741.

(53) Wang, H.; Liu, X. Y.; Chuah, Y. J.; Goh, J. C. H.; Li, J. L.; Xu, H. Design and engineering of silk fibroin scaffolds with biomimetic hierarchical structures. Chem. Commun. 2013, 49, 1431-1433.

(54) McDowall, A. W.; Chang, J. J.; Freeman, R.; Lepault, J.; Walter, C. A.; Dubochet, J. Electron microscopy of frozen hydrated sections of vitreous ice and vitrified biological samples. J. Microsc. 1983, 131, 1-9. 